\title{
A Hybrid Swarm Optimization Approach for Document Binarization
}

\author{
Mohamed ABD ELFATTAH ${ }^{1,2}$, Aboul Ella HASSANIEN ${ }^{3 *}$, Sherihan ABUELENIN ${ }^{1}$ \\ ${ }^{1}$ Computer Science Department, Faculty of Computers and Information, Mansoura University, 35516, Egypt \\ mohabdelfatah8@gmail.com,dr.sherihan@yahoo.com \\ ${ }^{2}$ Misr Higher Institute for Commerce and Computers, MET, Mansoura, Egypt \\ ${ }^{3}$ Faculty of Computers and Information, Cairo University, Egypt \\ aboitcairo@gmail.com (*Corresponding author)
}

\begin{abstract}
The binarization process is the preliminary and most significant phase of the document image analysis applications. A hybrid approach based on the merger of Salp swarm algorithm and the chaos theory is introduced. The proposed hybrid approach has been used to evaluate their ability and precision in the clustering process. It is revealed how Salp can operate to find automatically the centroid of a defined number of clusters using K-means objective function. Several different chaotic maps are integrated to adjust the behavior of the Salps by calibrating their random numbers. The efficiency of the proposed chaotic Salp swarm algorithm is empirically verified on the Document Image Binarization Contest H-DIBCO 2016 dataset. A comparison made between the proposed approach and some of the state-of-the-art methods in terms of F-Measure, Peak Signal to Noise Ratio and pseudo-F-Measure. In addition, Geometric-mean pixel accuracy, Distance Reciprocal Distortion Metric, Negative Rate Metric and Misclassification Penalty Metric are shown and discussed.
\end{abstract}

Keywords: K-means, Salp swarm Algorithm, Optimization, Clustering, H-DIBCO 2016.

\section{Introduction}

The process of classifying an unlabeled dataset into clusters according to their similarity, is called data clustering. It is considered the essential process of many applications such as stock prediction, signal processing, text mining, web application, pattern recognition and image segmentation (Jain, 2010). Recently, a huge amount of data has been made available for different fields such as medical, marketing, business, social science, and signal processing sectors. This data needs to be stored and retrieve easily (Serapião et al., 2016). Clustering process is considered the most important process in data mining, document retrieval, and pattern recognition, with huge data generated every day in different fields as digital imaging, video surveillance and Internet search. This data could be of various types including videos, images, and texts. Clustering process is a very critical process to perfectly classify these types of data and has proven its ability in different fields as statistics, pattern recognition, and machine learning (Jain, 2010), (Serapião et al., 2016).

Recently, Swarm Intelligence (SI) optimization algorithms have attracted many researchers in different fields. SI is one type of meta-heuristic optimization algorithms which depend on mimicking the behavior of birds, fish, and whale etc. Basically, different types of SI are presented and proposed in order to be involved in multiple areas such as; image processing, bioinformatics, wireless sensor networks (WSNs) and medical applications etc. The main target of these algorithms is to explore the search space for reaching the optimal solution that maximizes or minimizes the used objective function (Hassanien and Emary, 2018).

In the specialized literature, the clustering process is considered an NP-hard problem (Drineas et al., 2004). K-means is considered the most common clustering algorithm due to its properties that is very simple and fast. However, it is trapped into local minima (Serapião et al., 2016). Recently, the clustering problem has been solved as an optimization problem. Hence, a lot of different versions of optimization algorithms are conducted for solving this problem. The main target of clustering based on these optimization algorithms is to minimize the objective function, avoid the disadvantage of the basic K-means algorithm and to find the best solution (Han et al., 2017). These algorithms are evaluated using the objective function which is used in the basic $\mathrm{K}$-means clustering algorithm. Euclidean distance is considered the most common distance measure between objects.

(Banharnsakun, Sirinaovakul and Achalakul, 2013), have presented a clustering approach using the best-so-far artificial bee colony (ABC) (Banharnsakun, Achalakul and Sirinaovakul, 2011). They proposed a new concept named 
multiple patrilines to enhance the variety of solutions and allow the calculations to be spread between several computing units. A modified version named the bird flock gravitational search algorithm (BFGSA) was proposed (Han et al., 2017) . This approach consists of three phases named, initialization, identification, and orientation change. Also, the authors used 13 datasets from the UCI Machine Learning Repository to test the proposed approach and compared its performance with the standard GSA, Particle Swarm Optimization (PSO), ABC, Firefly Algorithm (FA), K-means, and four clustering algorithms. The results showed that the BFGSA can be employed effectively for data clustering.

(Abualigah et al., 2017) introduced a hybrid version of krill herd $(\mathrm{KH})$ and harmony search (HS), named (H-KHA) for data clustering. Seven benchmark datasets from UCI and six common text datasets are employed to assess the proposed approach. In addition, it is compared with different well-known clustering methods and other optimization algorithms. The results reveal that it is suitable for data and text clustering.

In (Serapião et al., 2016), Fish School Search (FSS) in multidimensional Data Clustering was introduced and compared with PSO in parallel and serial processing based on the integration with the K-means and K-harmonic means (KHM). 13 benchmark datasets were used to evaluate these approaches. The results proved that using FSS for clustering is a promising procedure.

(Khalaf et al., 2018), presented a new methodology for overcoming the shortness of the basic FA, by introducing a new weighted $\mathrm{K}$-means with an enhanced version of FA named (WKIFA). An application of indexing a large iris database and selected dataset from UCI are employed to assess the approach. The WKIFA is better than K-means.

According to the No Free Lunch theorem (NFL) (Wolpert and Macready, 1997), no single algorithm performed well in all of the problems or in other words, none of them solves all problems. The evaluation of the performance of the integration of chaos maps with the Salp Swarm Algorithm (Mirjalili et al., 2017), which has been recently introduced, is encouraged to be achieved. It is based on the behavior of Salps when navigating and foraging in oceans and it is mainly used to solve different kinds of problems as in (Sayed, Khoriba and Haggag, 2018), (Faris et al., 2018), (El-Fergany, 2018). The main aim of this paper is to use chaos maps with the Salp Swarm Algorithm to present a chaotic version of SSA algorithm for document binarization which minimizes the objective function of the standard k-means (CSSAK). This novel hybrid algorithm tries to produce a high-quality binarized image of old manuscripts. The basic contributions of this paper could be summarized as follow:

- Three chaotic versions of the SSA algorithm are introduced for the document binarization based k-means objective function, named (CSSAK) and the best one is chosen.

- The H-DIBCO 2016 dataset is employed to test the CSSAK versions.

- Ten chaos maps are employed to tune the behavior of salp in the CSSAK.

The remaining paper is structured as follows; Section 2 presents the basics of Salp Swarm Algorithm (SSA) and introduces the chaotic theory. The proposed approach is introduced in Section 3. In Section 4, the experimental results and discussion are given. Finally, conclusion and future work are clarified in Section 5.

\section{Preliminaries}

This section introduces the basics of Salp Swarm Algorithm (SSA) and a brief introduction regarding the chaotic theory, including, details of each chaos map with a mathematical explanation.

\subsection{Salp Swarm Algorithm (SSA)}

\subsubsection{Inspiration}

SSA (Mirjalili et al., 2017) is considered one of the recently introduced meta-heuristic algorithms, that simulated the behavior of salps in oceans. Salps, a type of Salpdae are very similar to jellyfish with respect to two features: tissue and movement. Salps form a swarm called Salp chain. Until now, this behavior is not clear. Some studies denoted to this behavior when the salps are seeking for getting the best locomotion depending on fast foraging and coordinated changes (Mirjalili et al., 2017), (Sayed, Khoriba and Haggag, 2018).

\subsubsection{Mathematical Model}

There are two types of Salps. The first one is named "the leader" and takes the first position on the chain, while the second one is named "the 
follower" and takes the remaining positions on the chain. The salp (leaders) update their position based on the following Equation 1 (Mirjalili et al., 2017).

$a_{j}^{1}= \begin{cases}F_{j}+g_{1}\left(\left(u b_{j}-l b_{j}\right) g_{2}+l b_{j}\right), & g_{3} \geq 0 \\ F_{j}-g_{1}\left(\left(u b_{j}-l b_{j}\right) g_{2}+l b_{j}\right), & g_{3}<0\end{cases}$

where $a_{j}^{1}$ indicates the position of the first salp (leader) in $j t h$ dimension, $l b_{i}$ and $u b_{i}$ are the lower and the upper bounds in $j$ th dimension. $g_{1}, g_{2}$ and $g_{3}$ are random numbers (Mirjalili et al., 2017), and $F_{j}$ is the position of the food source. $g_{1}$ is considered important because it is responsible for the balance between the exploration and exploitation in SSA. It is computed as follows:

$g_{1}=2 e^{-\left(\frac{4 h}{H}\right)^{2}}$

where $h$ and $H$ denote the current iteration and the maximum number of iterations, respectively. $g_{2}$ and $g_{3}$ are random numbers in the interval $[0,1]$. They are used for determining if the next position should be towards positive infinity or negative infinity in the $j t h$ dimension. The followers of the leader update their position based on the following Equation 3, which uses Newton's law of motion (Mirjalili et al., 2017).

$a_{j}^{i}=\frac{1}{2} n t^{2}+q_{0} t$

where $a_{j}^{i}$ denotes the position of the followers in $j$ th dimension, and $i \geq 2, q_{0}$ is the initial speed, $n=\frac{q_{\text {final }}}{q_{0}}$ where $q=\frac{a-a_{0}}{t}$, and $t$ represents the time. Time is considered in optimization as an iteration (Mirjalili et al., 2017), so, the discrepancy among iterations equals 1 . If $q_{0}=0$ then Equation 3 could be represented as follows:

$a_{j}^{i}=\frac{1}{2}\left(a_{j}^{i}+a_{j}^{i-1}\right)$

where $i \geq 2$, and $a_{j}^{i}$ denotes the position of the followers $i t h$ in $j$ th dimension (detailed in (Mirjalili et al., 2017)).

\subsection{Chaotic Theory}

The properties of chaotic systems have been implemented in different sciences as economy, biology, medicine, engineering, and others (Tavazoei and Haeri, 2007). Chaos is precisely expressed as randomness created by simple deterministic systems. The chaos maps have a lot of advantages (Tavazoei and Haeri, 2007), ergodicity, the sensitive dependence on initial conditions, and the semi-stochastic property. These advantages make the chaos reach the best solution faster than the stochastic search (Hassanien and Emary, 2018). So, the randomness of the basic SSA is replaced with chaotic dynamics, therefore, three chaotic versions of the SSA are introduced and tested, where ten different non-invertible maps with various properties are used. Where $E_{t}$ indicates to $t$-th number of the chaotic sequence, $t$ is the index of the chaotic sequence $E$, while, other parameters $b, \mu, a$ are the control parameters that used to tune the chaotic behavior of the dynamic systems, the chaos range as in (Sayed, Khoriba and Haggag, 2018). The mathematical equations of the used chaos maps given below.

\section{Chaos map 1: Chebyshev}

$$
E_{t+1}=\cos \left(t \cos ^{-1}\left(E_{t}\right)\right)
$$

2. Chaos map 2: Sinusoidal

$E_{t+1}=a E_{t}^{2} \sin \left(\pi E_{t}\right)$

where $\mathrm{a}=2.3$.

3. Chaos map 3: Logistic

$E_{t+1}=a E_{t}\left(1-E_{t}\right)$

where $a=4$.

\section{Chaos map 4: Gauss/mouse}

$E_{t+1}= \begin{cases}1, & E_{t}=0 \\ \frac{1}{\bmod \left(E_{t}, 1\right)}, & \text { otherwise }\end{cases}$

\section{Chaos map 5: Piecewise}

$$
E_{t+1}=\left\{\begin{array}{ll}
\frac{E_{t}}{P}, & 0 \leq E_{t} \leq P \\
\frac{E_{t}-P}{0.5-P}, & P \leq E_{t}<\frac{1}{2} \\
\frac{1-P-E_{t}}{0.5-P}, & \frac{1}{2} \leq E_{t}<1-P \\
\frac{1-E_{t}}{P}, & 1-P \leq E_{t}<1
\end{array}, P=0.4\right.
$$

\section{Chaos map 6: Sine map}

$$
E_{t+1}=\frac{a}{4} \sin \left(\pi E_{t}\right)
$$

where $\mathrm{a}=4$. 
7. Chaos map 7: Tent map

$$
E_{t+1}= \begin{cases}\frac{E_{t}}{0.7}, & E_{t}<0.7 \\ \frac{10}{3}\left(1-E_{t}\right), & E_{t} \geq 0.7\end{cases}
$$

\section{Chaos map 8: Circle map}

$$
E_{t+1}=\bmod \left(E_{t}+b-\left(\frac{a}{2 \pi}\right) \sin \left(2 \pi E_{t}\right), 1\right)
$$

where $a=0.5$ and $b=0.2$.

\section{Chaos map 9: Singer}

$E_{t+1}=\mu\left(7.86 E_{t}-23.31 E_{t}^{2}+28.75 E_{t}^{3}-13.302875 E_{t}^{4}\right)$

where $\mu=1.07$

\section{Chaos map 10: Iterative}

$$
E_{t+1}=\sin \left(\frac{a \pi}{E_{t}}\right)
$$

where $\mathrm{a}=0.7$

\section{The Proposed Approach}

In this paper, three chaotic versions of the SSA are introduced and compared for the document binarization. These versions are based on each of the parameters $\left(g_{1}, g_{2}\right.$ and $\left.g_{3}\right)$ of the SSA as mentioned in Equation 1 and adjusted individually according to the chaotic maps. The three chaotic versions of the SSA are abbreviated as, CSSAKI that based on the tuning the first parameter, CSSAKII that depends on the tuning the second parameter, finally is CSSAKIII, that based on the tuning the third parameter.

\subsection{SSAK, CSSAK Versions Parameters Setting}

The parameter setting of all optimization algorithms is considered the main step to reach the optimal solution. So, salps positions of SSAK and CSSAK algorithms are initialized randomly, the number of population size (search agents) is set to 8 , the maximum iterations number is 20 , the upper bounds and lower bounds are set in the interval [0 255].

\subsection{Fitness Function}

The clustering problem could be summarized as follows: The input is a data set of patterns in $d$ -dimensional space, is partitioned based on the predefined number of $k$ clusters. The SSA can handle the clustering problems in such a way that each salp (search agent) holds centroids of clusters and tries to find the most feasible cluster centroids for getting best clustering option. At each iteration, each salp position is assessed using the following function (15)

$J=\sum_{j=1}^{k} \sum_{i=1}^{m}\left\|x_{i}^{(j)}-c_{j}\right\|^{2}$

where $\left\|x_{i}^{(j)}-c_{j}\right\|^{2}$ denotes the distance between the cluster center $c_{j}$ and a data point $x_{i}^{j}$, is considered an indication of the distance of the $m$ data points from the related cluster centers, whose main objective is to minimize this function (MacQueen et al., 1967).

\subsection{Position Updating (Optimization)}

Salp's position is evaluated at each iteration based on the selected objective function 15. Subsequently, the best position of salps is picked out. Then every salp (follower) individually updates its position in the search space according to the best (leader) position, hence, the binary image is created based on the optimal solution that CSSAK reaches it. The output of this phase is the cluster centers, which are used to create the black and white image (binary image), the cluster centers are updated at each iteration, a good balance between exploration and exploitation of CSSAK during this phase will reach the optimal solution, hence, the high-quality binarized image is produced.

\subsection{Termination Criteria}

The proposed algorithm CSSAK is completed when it reaches the optimal (best) solution or the maximum number of iterations.

\section{Experimental Results and Discussion}

The experiments were conducted in order to assess the influence of the chaos maps over the basic SSA algorithm for the document binarization given below.

\subsection{Experiments Setup}

The three variant chaotic versions of SSA are implemented on a PC using Windows 7, and Intel 
Core i5-2410 M processor with 4GB in RAM on MATLAB R2018a.

\subsection{Datasets}

The H-DIBCO 2016 (Pratikakis et al., 2016) dataset is used to test the proposed approach, it contains 10 different handwritten documents images with ground truth among which this is publicly available. Figure 1 illustrates samples of this dataset. Some of the problems related to these images as faded ink, poor quality, bleed-through and stains are shown. Hence, these problems are making the binarization process of that type an open challenge.

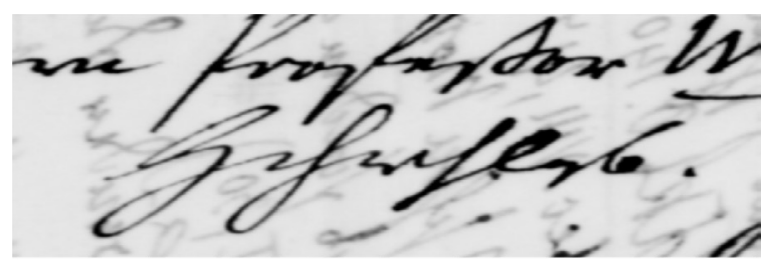

(a)

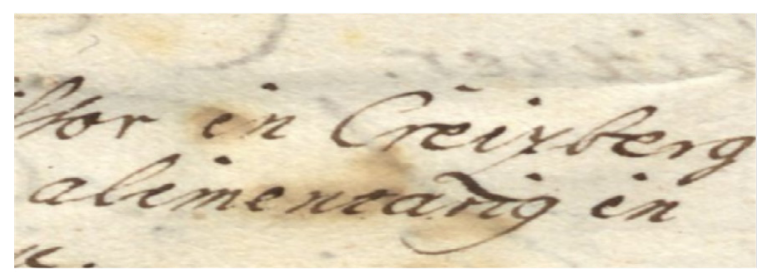

(b)

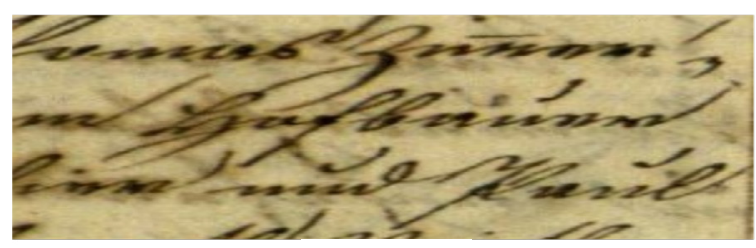

(c)

Figure 1. Sub-images (data from Pratikakis et al., 2016), H-DIBCO 2016

\subsection{Performance Measures}

In this paper, the H-DIBCO 2016 (Pratikakis et al., 2016) contest performance measures criteria were followed. Table 1 shows the performance measures used to evaluate all chaotic versions of the SSA. As it appears in the last column two arrows are used, the first one $(\uparrow)$ indicates better result when the value of the measure is high in the following measure (FM, p-FM, PSNR, and GA), the second one $(\downarrow)$ is better when their values are low in (DRD, NRM, and MPM). According to (Paredes, Kavallieratou and Lins, 2010), the binarization problem is an unbalanced problem. So, the geometric-mean pixel accuracy (GA) has been used as an additional performance measure. The source code used is available in (Moghaddam and Nafchi, 2013).

Table 1. Performance measures

\begin{tabular}{|c|c|c|}
\hline \multirow[t]{2}{*}{$\begin{array}{l}\text { Performance } \\
\text { measure }\end{array}$} & \multirow[t]{2}{*}{ 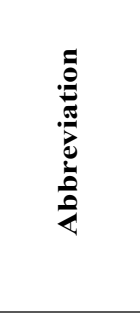 } & 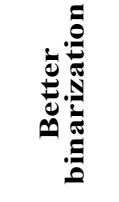 \\
\hline & & $\begin{array}{l}\operatorname{Max}(\eta) / \\
\operatorname{Min}(\emptyset)\end{array}$ \\
\hline $\begin{array}{c}\text { F-Measure } \\
\text { (Sokolova and Lapalme, 2009) }\end{array}$ & F-Measure & $(\uparrow)$ \\
\hline $\begin{array}{c}\text { pseudo F Measure } \\
\text { (Ntirogiannis, Gatos and Pratikakis, } \\
\text { 2008) (Pratikakis, Gatos and } \\
\text { Ntirogiannis, 2010) }\end{array}$ & p-FM & $(\uparrow)$ \\
\hline $\begin{array}{l}\text { Peak Signal to Noise Ratio } \\
\text { (Pratikakis et al., 2016) }\end{array}$ & PSNR & $(\uparrow)$ \\
\hline $\begin{array}{c}\text { Geometric-mean pixel } \\
\text { accuracy (Paredes, Kavallieratou } \\
\text { and Lins, 2010) }\end{array}$ & GA & $(\uparrow)$ \\
\hline $\begin{array}{c}\text { Distance Reciprocal Distortion } \\
\text { Metric } \\
\text { (Lu, Kot and Shi, 2004) }\end{array}$ & DRD & ( $\downarrow)$ \\
\hline $\begin{array}{l}\text { Negative Rate Metric (Pratikakis, } \\
\text { Gatos and Ntirogiannis, 2010) }\end{array}$ & NRM & ( $\downarrow)$ \\
\hline $\begin{array}{l}\text { Misclassification Penalty } \\
\text { Metric (Young and Ferryman, 2005) }\end{array}$ & MPM & ( $)$ \\
\hline
\end{tabular}

\subsection{Results and Discussion}

This section is designed to introduce the results of the basic SSAK, and three chaotic versions CSSAKI, CSSAKII, CSSAKIII algorithms, both of, the numerical results, and the convergence curves are used in this comparison, this comparison is done using the same dataset, and the performance measures (see Sections $4.2,4.3)$. Finally, the best chaotic version is chosen as a recommended chaotic version of SSAK. In addition to, the chosen (best) one is compared with the basic SSAK under the same parameters, as well as, the submitted methods to the H-DIBCO 2016 contest, the well-known binarization methods (Otsu, Sauvola) and the recent binarization methods.

\subsubsection{The Effect of the Chaos Maps of the CSSAK Versions}

In this subsection, each parameter of the original SSA is tuned by the chaotic maps. So, 
Table 2. Detailed results of the CSSAKI, CSSAKII, and CSSAKIII using H-DIBCO 2016 dataset.

\begin{tabular}{|c|c|c|c|c|c|c|c|c|}
\hline Chaos map name & $\begin{array}{l}\text { CSSAK } \\
\text { versions }\end{array}$ & F-measure & p-FM & PSNR & GA & DRD & NRM & МРМ \\
\hline \multirow{3}{*}{ Chebyshev map } & CSSAKI & 90.43 & 92.91 & 19.05 & 0.94 & 3.64 & 5.79 & 1.99 \\
\hline & CSSAKII & 86.41 & 89.67 & 17.32 & 0.91 & 5.08 & 7.85 & 3.16 \\
\hline & CSSAKIII & 85.93 & 88.45 & 17.38 & 0.94 & 7.11 & 6.32 & 7.36 \\
\hline \multirow{3}{*}{ Circle map } & CSSAKI & 89.30 & 91.63 & 18.47 & 0.93 & 4.08 & 6.18 & 1.86 \\
\hline & CSSAKII & 86.48 & 90.35 & 17.38 & 0.92 & 6.05 & 6.90 & 4.21 \\
\hline & CSSAKIII & 85.93 & 88.57 & 17.18 & 0.93 & 7.24 & 6.06 & 4.33 \\
\hline \multirow{3}{*}{ Gauss/mouse map } & CSSAKI & 89.17 & 92.36 & 18.55 & 0.93 & 4.01 & 7.15 & 1.71 \\
\hline & CSSAKII & 86.07 & 90.00 & 17.40 & 0.91 & 5.54 & 7.46 & 3.07 \\
\hline & CSSAKIII & 84.99 & 87.23 & 16.39 & 0.94 & 7.66 & 5.15 & 5.76 \\
\hline \multirow{3}{*}{ Iterative map } & CSSAKI & 89.03 & 91.55 & 18.30 & 0.94 & 4.28 & 6.34 & 2.30 \\
\hline & CSSAKII & 85.41 & 88.17 & 17.32 & 0.91 & 5.98 & 7.65 & 5.66 \\
\hline & CSSAKIII & 87.02 & 89.22 & 17.27 & 0.93 & 5.69 & 5.62 & 2.92 \\
\hline \multirow{3}{*}{ Logistic map } & CSSAKI & 88.82 & 91.87 & 18.16 & 0.93 & 4.81 & 5.77 & 2.02 \\
\hline & CSSAKII & 85.32 & 88.69 & 17.07 & 0.91 & 5.92 & 8.04 & 4.47 \\
\hline & CSSAKIII & 86.38 & 89.22 & 17.31 & 0.92 & 5.26 & 7.01 & 2.79 \\
\hline \multirow{3}{*}{ Piecewise map } & CSSAKI & 89.24 & 92.34 & 18.48 & 0.93 & 4.04 & 6.42 & 2.35 \\
\hline & CSSAKII & 87.19 & 91.61 & 18.12 & 0.89 & 4.31 & 9.24 & 1.36 \\
\hline & CSSAKIII & 87.53 & 90.47 & 17.54 & 0.93 & 5.31 & 5.97 & 3.49 \\
\hline \multirow{3}{*}{ Sine map } & CSSAKI & 89.48 & 91.93 & 18.51 & 0.94 & 4.25 & 5.79 & 2.93 \\
\hline & CSSAKII & 85.96 & 90.12 & 17.60 & 0.90 & 5.02 & 8.69 & 2.45 \\
\hline & CSSAKIII & 86.04 & 89.06 & 17.74 & 0.91 & 5.87 & 7.29 & 4.57 \\
\hline \multirow{3}{*}{ Singer map } & CSSAKI & 88.99 & 92.57 & 18.52 & 0.92 & 3.97 & 7.58 & 1.57 \\
\hline & CSSAKII & 87.43 & 91.42 & 18.10 & 0.91 & 4.47 & 8.50 & 1.69 \\
\hline & CSSAKIII & 87.37 & 91.31 & 18.09 & 0.90 & 4.40 & 8.61 & 1.55 \\
\hline \multirow{3}{*}{ Sinusoidal map } & CSSAKI & 89.03 & 92.59 & 18.46 & 0.92 & 4.05 & 6.98 & 1.86 \\
\hline & CSSAKII & 84.68 & 89.99 & 17.73 & 0.88 & 5.15 & 10.85 & 1.81 \\
\hline & CSSAKIII & 85.44 & 89.12 & 17.02 & 0.91 & 6.32 & 7.59 & 6.19 \\
\hline \multirow{3}{*}{ Tent map } & CSSAKI & 89.33 & 92.01 & 18.52 & 0.94 & 4.20 & 6.10 & 2.15 \\
\hline & CSSAKII & 87.63 & 90.82 & 17.99 & 0.92 & 4.83 & 6.96 & 2.15 \\
\hline & CSSAKIII & 86.91 & 88.96 & 17.25 & 0.94 & 5.91 & 4.94 & 4.63 \\
\hline
\end{tabular}

in the following three chaotic versions named CSSAKI, CSSAKII and CSSAKIII are tested and evaluated based on the H-DIBCO 2016 dataset. The detailed results of the chaos map effect on each parameter are presented in Table 2 which illustrates the performance measures values of this experiment. The value of these measures of the CSSAKI- Chebyshev map named F-measure, p-FM, PSNR, DRD was much better than other CSSAK versions, except for the NRM, and MPM values. The CSSAKIII-Tent is the best on the NRM with the value of (4.94), the CSSAKII- Piecewise is the best on the MPM with the value of (1.36), while some other versions are equal to the GA measure with value (0.94) as illustrated in Table 2 . The results from Table 2 could be summarized as follows:

- The first parameter $g_{1}$ with the chaos maps has a great effect on tuning the behavior of the CSSAKI algorithm, so, it produced the best result in most of the performance measures.

- Analyzing the values of the GA measure, it can be noticed that most of the chaotic versions have values higher than 0.90 , which indicates that they are able to produce a binary image with a lower classification error between the black and white pixels.

- Basically, some of the chaotic versions are close together in MPM measure, that produces binarized images with high-quality and low misclassified pixels like the ground truth images.

\subsubsection{Convergence Curves of the Chaotic Versions}

The convergence curves of the different chaotic versions of the SSA algorithm are discussed and analyzed in this subsection. Some convergence curves are picked out through the different runs. Figure 2 illustrates the convergence curves. As shown in this Figure, some remarks could be concluded as follows:

- In Figures 2-a, b, and c, the CSSAKIChebyshev, CSSAKI-Circle, and CSSAKIGuass/mouse obtained the best result and converged faster than the other chaotic versions to the optimal solution. 
Table 3. Detailed results of the CSSAKI- Chebyshev and SSAK using H-DIBCO 2016 dataset

\begin{tabular}{|c|c|c|c|c|c|c|c|}
\hline Approach name & F-measure & p-FM & PSNR & GA & DRD & NRM & MPM \\
\hline SSAK & 86.26 & 89.28 & 17.33 & 0.91 & 5.22 & 7.60 & 2.10 \\
\hline CSSAKI-Chebyshev & $\mathbf{9 0 . 4 3}$ & $\mathbf{9 2 . 9 1}$ & $\mathbf{1 9 . 0 5}$ & $\mathbf{0 . 9 4}$ & $\mathbf{3 . 6 4}$ & $\mathbf{5 . 7 9}$ & $\mathbf{1 . 9 9}$ \\
\hline
\end{tabular}

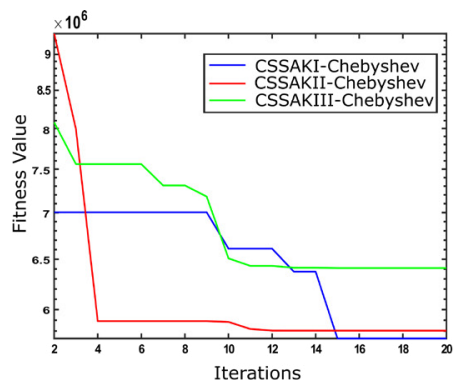

(a)

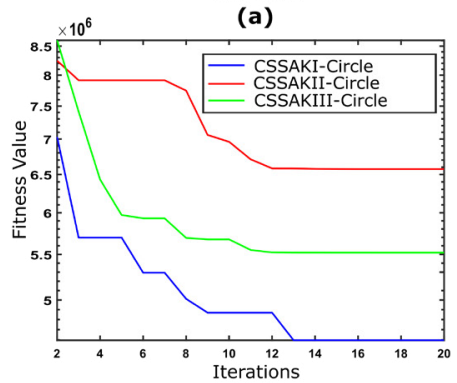

(b)

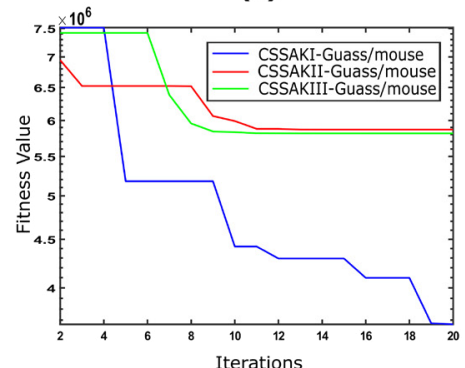

(c)

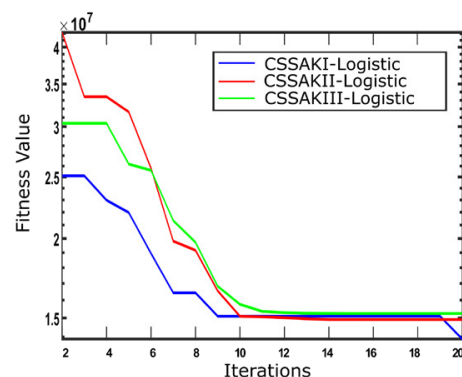

(d)

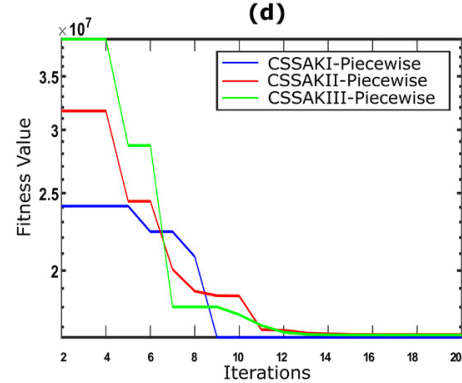

(e)

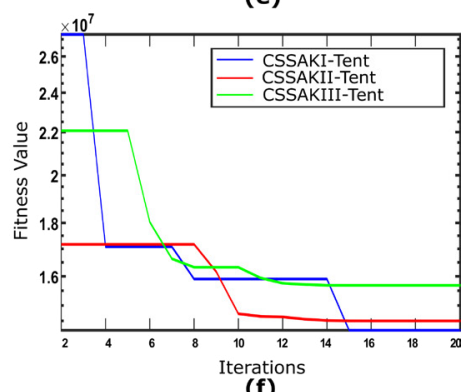

(f)

Figure 2. Convergence curves of the CSSAKI, CSSAKII and CSSAKIII

- The convergence curves that displayed in Figure 2-d show that the CSSAKI-Logistic map is converged faster than other versions while the CSSAKIII-Logistic achieved the worst result.

- The convergence curves of the three versions of CSSAK-Piecewise illustrated in Figure 2-e, being obvious that CSSAKI-Piecewise reached faster while the CSSAKII-Piecewise and CSSAKIII-Piecewise are reached nearly the same time.

- Figure 2-f illustrates the convergence curves of the CSSAK-Tent. The CSSAKI-Tent is the best one and CSSAKII-Tent obtained the second-best results, while the worst result is provided by the CSSAKIII-Tent.

To conclude, the CSSAKI- Chebyshev is the best chaotic version of the SSA algorithm. Therefore, the Chebyshev map was chosen as a suitable map to form the best chaotic SSA (CSSAK) that will be compared with the basic SSAK and other binarization methods (see subsections 4.5, 4.7).

\subsection{Comparison with the Bbasic SSAK Algorithm}

This experiment was designed to evaluate the influence of the chaos map on the SSA algorithm. In this experiment, for both algorithms, i.e. SSAK and CSSAKI-Chebyshev, the comparison was made under the same SSA parameters, datasets, and performance measures (see subsections 4.2, and 4.3). Furthermore, the convergences curves were drawn and analyzed. Table 3 illustrates the detailed numerical result, (the best results are put in bold). The CSSAKI-Chebyshev performs better than the basic SSAK and had the best results among all the performance measures. During the optimization 
phase, some convergence curves between them are chosen. Figure 3 illustrates the convergence curves of the SSAK and CSSAKI-Chebyshev. Some remarks could be concluded as follows:

- In Figures 3-a, b, and c, can easily be noticed that the CSSAKI-Chebyshev reached the optimal solution faster than the SSAK.

- In Figure 3-d, it can be remarked that the CSSAKI-Chebyshev and the SSAK reached the optimal solution approximately at the same time. However, the CSSAKI-Chebyshev reached the solution faster than SSAK.
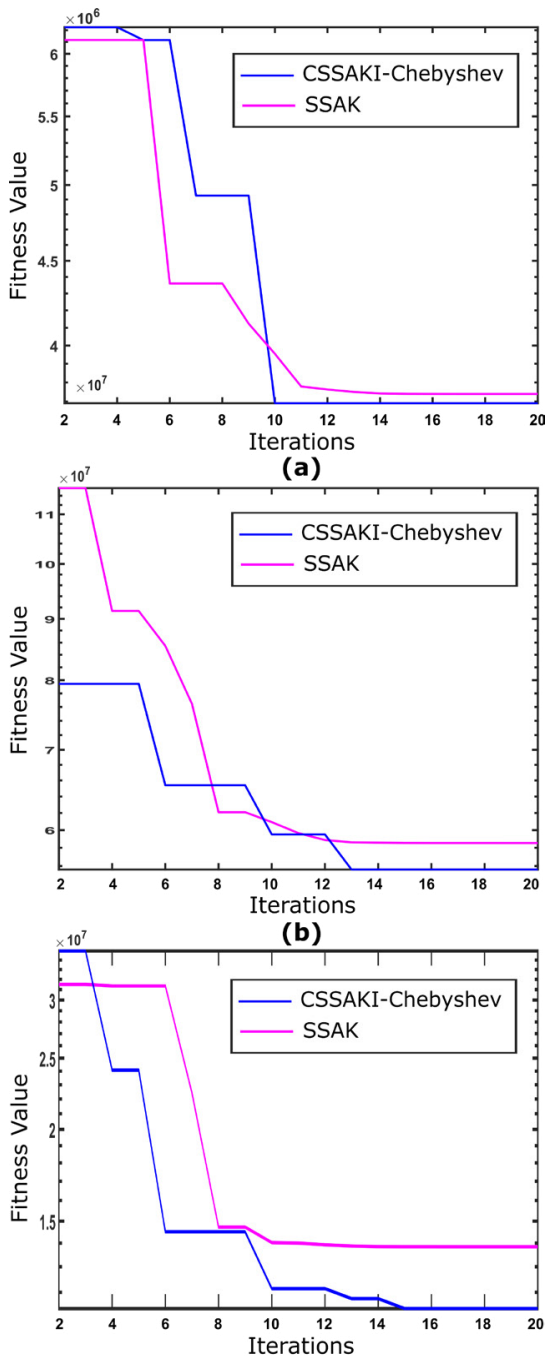

(c)

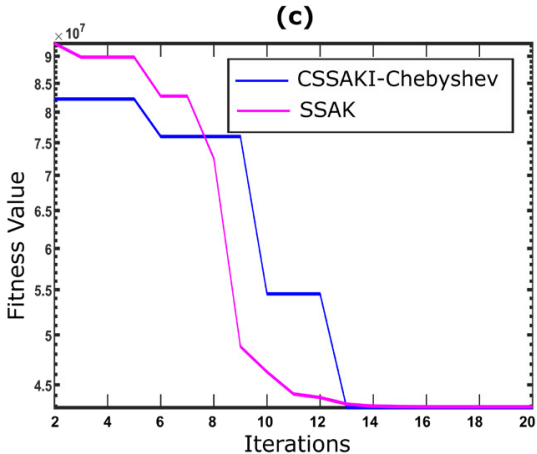

(d)

Figure 3. Convergence curves of the CSSAKIChebyshev and SSAK
It can be concluded that the effect of the chaos map results in the improvement of the SSAK algorithm with the aim of helping the basic SSAK to increase its ability to find the optimal solution faster.

\subsection{Visual Inspection Analysis the CSSAKI- Chebyshev}

This experiment is used to evaluate the output image from the CSSAKI- Chebyshev. As shown in Figure 4, four images are picked out through the optimization process, that presented the best solution, to show the effective role of the proposed approach in producing a binarized document with a complete character structure. Therefore, in this Figure, it could be noticed that the original images shown in Figure 4-a are subject to various types of noise, that made obtaining high-quality binarized images a very difficult task. The ground truth images are illustrated in Figure 4-b. The binarized images as illustrated in Figure 4-c, are very similar to the ground truth (GT) image. The best binarized image is achieved in the second row, which contains the least noise, the text and background are very close to the GT image. We could conclude that the proposed approach produces a good binary image.

\subsection{Comparison with the State-of-arts Methods}

The purpose of this subsection is to compare the result of the CSSAKI-Chebyshev algorithm with the best four methods submitted to H-DIBCO 2016 contest (detailed in (Pratikakis et al., 2016)), as well as the recent binarization algorithms. Table 4 shows the numerical results of these methods in terms of the used performance measures. The proposed approach obtained the best result in comparison with all methods mentioned submitted to H-DIBCO 2016 contest and in comparison with all the wellknown binarization methods (Otsu, and Sauvola), according to this comparison, the proposed approach achieved the best result in MPM measure with a value of (1.99), and obtained the second best result in terms of the F measure, p-FM and PSNR, DRD with values of 90.43, $92.91,19.05$, and 3.64, respectively.

\subsection{Discussion}

This discusses and offers an explanation regarding the CSSAK results, as they were shown in the previous sections. It was found that the CSSAKI- 

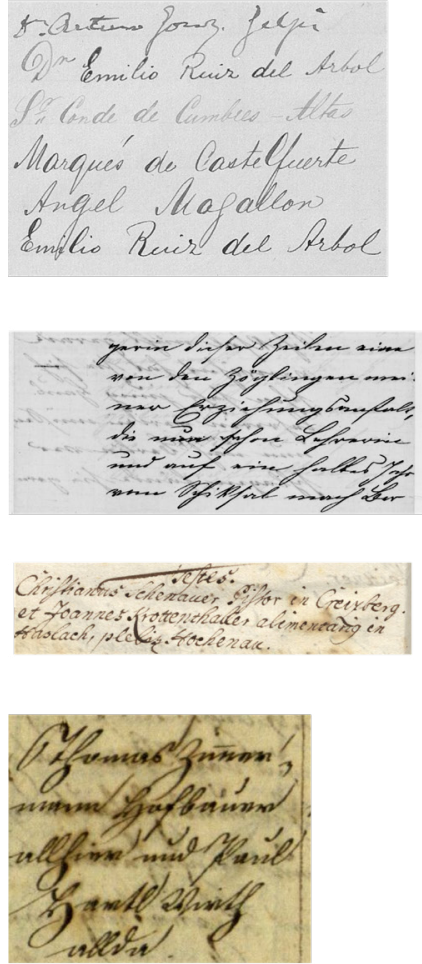

(a)

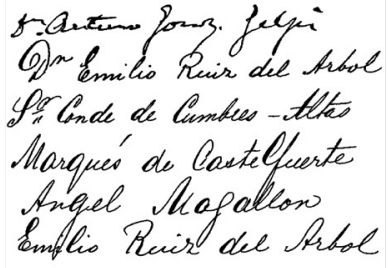

santers Pons. Jogi

Oj) Emilio Ruir del Arbol

for lande de limber - thtes

Marqué de Castelfuerte.

Anquel Lagallon

omflio Ruir del Arbol
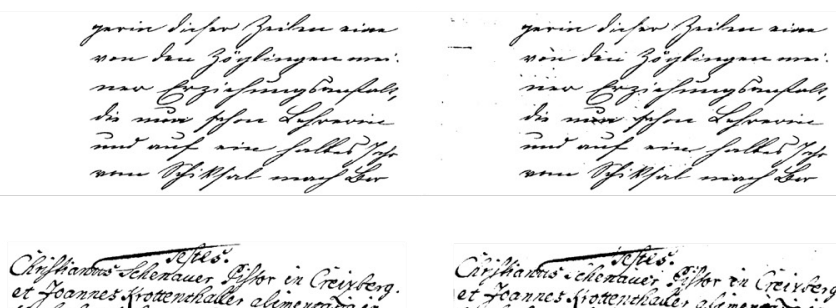

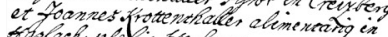

Givition

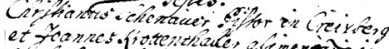

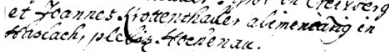

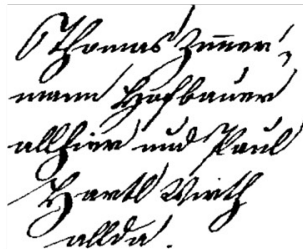

(b)

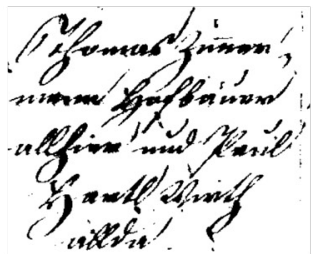

(c)

Figure 4. a) Original images selected from H-DIBCO 2016, b) Ground truth images provided by H-DIBCO 2016, and c) Results of the proposed approach

Table 4. CSSAKI- Chebyshev vs. binarization methods using H-DIBCO 2016 dataset

\begin{tabular}{|c|c|c|c|c|c|c|}
\hline Approach Name & F-measure & p-FM & PSNR & DRD & NRM & MPM \\
\hline Nati Kligler et al. (Pratikakis et al., 2016) & 87.61 & - & 18.11 & 5.21 & - & - \\
\hline Yazid Hassaine et al.3 (Pratikakis et al., 2016) & 88.72 & - & 18.45 & 3.86 & - & - \\
\hline Yazid Hassaine et al.2 (Pratikakis et al., 2016) & 88.47 & - & 18.29 & 3.93 & - & - \\
\hline Edward Roe et al. (Pratikakis et al., 2016) & 87.97 & - & 18.00 & 4.49 & - & - \\
\hline Otsu (Otsu, 1979) (Pratikakis et al., 2016) & 86.61 & - & 17.80 & 5.56 & - & - \\
\hline $\begin{array}{c}\text { Sauvola (Sauvola and Pietikäinen, 2000) } \\
\text { (Pratikakis } \text { et al., 2016) }\end{array}$ & 82.52 & - & 16.42 & 7.49 & - & - \\
\hline $\begin{array}{c}\text { Mitianoudis (Mitianoudis and Papamarkos, } \\
\text { 2015) (Jia } \text { et al., 2018) }\end{array}$ & 86.89 & - & 17.60 & - & $\mathbf{1 . 7 6}$ & - \\
\hline $\begin{array}{c}\text { Lelore (Lelore and Bouchara, 2013) } \\
\text { (Jia } \text { et al., 2018) }\end{array}$ & 87.21 & 88.48 & 17.36 & 5.27 & 4.93 & 7.86 \\
\hline Nhat Vo et al. (Vo et al., 2018) & 90.10 & - & 19.01 & $\mathbf{3 . 5 8}$ & - & - \\
\hline Jia et al. a (Jia et al., 2016) & 85.56 & 91.24 & 17.57 & 6.11 & 9.32 & 10.91 \\
\hline Jia et al. b (Jia et al., 2018) & $\mathbf{9 0 . 4 8}$ & $\mathbf{9 3 . 2 7}$ & $\mathbf{1 9 . 3 0}$ & 3.97 & 4.95 & 6.90 \\
\hline \begin{tabular}{c} 
CSSAKI- Chebyshev \\
\hline
\end{tabular} & 90.43 & 92.91 & 19.05 & 3.64 & 5.79 & $\mathbf{1 . 9 9}$ \\
\hline
\end{tabular}

Chebyshev method can converge towards the optimal solution faster than the SSAK. Moreover, it produced the better-binarized image for both the numerical results and the visual inspection. The proposed approach result is consistent with most of the performance measure, as illustrated by the results compared in Tables 2, 3, and 4 .
It can be remarked that all chaotic versions based on the first parameter are better than other chaotic versions and all the methods mentioned submitted to H-DIBCO 2016 contest in most of the performance measures values. Studying the influence of the role of the chaos maps for adjusting the basic SSA random numbers 
algorithm produces high-quality binarized images. It is worth mentioning, that the proposed approach results are obtained without training or post-processing phase, the chaos maps can achieve an excellent balance between the exploration and exploitation phases during the optimization phase that obtained the best cluster centers positions, hence, the minimum value of the objective function is obtained, therefore, a high-quality binarized image is construct.

\section{Conclusion and Future Work}

This paper introduced three chaotic versions of the SSA algorithm for the document binarization. The three random parameters of the SSA are improved based on the well-known ten chaos maps. In this paper, the first experiment was carried out in order to select the best chaotic version through

\section{REFERENCES}

1. Abualigah, L. M. et al. (2017). A novel hybridization strategy for krill herd algorithm applied to clustering techniques, Applied Soft Computing, 60, 423-435. Elsevier.

2. Banharnsakun,A.,Achalakul, T.\& Sirinaovakul, B. (2011). The best-so-far selection in artificial bee colony algorithm, Applied Soft Computing, 11(2), 2888-2901. Elsevier.

3. Banharnsakun, A., Sirinaovakul, B. \& Achalakul, T. (2013). The best-so-far ABC with multiple patrilines for clustering problems, Neurocomputing, 116, 355-366. Elsevier.

4. Drineas, P. et al. (2004). Clustering large graphs via the singular value decomposition, Machine Learning, 56(1-3), 9-33. Springer.

5. El-Fergany, A. A. (2018). Extracting optimal parameters of PEM fuel cells using Salp Swarm Optimizer, Renewable Energy, 119, 641-648. Elsevier.

6. Faris, H. et al. (2018). An efficient binary Salp Swarm Algorithm with crossover scheme for feature selection problems, KnowledgeBased Systems, 154, 43-67. Elsevier.

7. Han, X. et al. (2017). A novel data clustering algorithm based on modified gravitational search algorithm, Engineering Applications of Artificial Intelligence, 61, 1-7. Elsevier. evaluating the influence of each chaos map to tune the three random numbers on the original SSA, the CSSAKI-Chebyshev algorithm obtained the best results among all the other chaotic versions. The second experiment was carried out in order to compare the CSSAKI-Chebyshev algorithm (the best chaotic version of SSA) with the basic SSAK. It was demonstrated that it performed better than SSAK. In the third experiment, the CSSAKIChebyshev was compared with the methods mentioned submitted to the H-DIBCO 2016 contest, the well-known binarization methods (Otsu, Sauvola), and the recent binarization methods. The results revealed that the proposed approach ranked first in the MPM measure. The proposed approach could be used to achieve high quality in the different areas in detecting the clusters on multi-dimensional dataset.

8. Hassanien, A. E. \& Emary, E. (2018). Swarm Intelligence: Principles, Advances, and Applications. CRC Press.

9. Jain, A. K. (2010). Data clustering: 50 years beyond K-means, Pattern Recognition Letters, 31(8), 651-666. Elsevier.

10. Jia, F. et al. (2016). Document image binarization using structural symmetry of strokes. In $201615^{\text {th }}$ International Conference on Frontiers in Handwriting Recognition (ICFHR) (pp. 411-416).

11. Jia, F. et al. (2018). Degraded Document Image Binarization using Structural Symmetry of Strokes, Pattern Recognition, 74, 225-240. Elsevier.

12. Khalaf,E.T.etal.(2018).Efficient Classifying and Indexing for Large Iris Database Based on Enhanced Clustering Method, Studies in Informatics and Control, 27(2), 191-202, DOI: $10.24846 / \mathrm{v} 27 \mathrm{i} 2 \mathrm{y} 201807$

13. Lelore, T. \& Bouchara, F. (2013). Fair: A fast algorithm for document image restoration, IEEE Transactions on Pattern Analysis and Machine Intelligence, 35(8), 2039-2048. IEEE.

14. Lu, H., Kot, A. C. \& Shi, Y. Q. (2004). Distance-reciprocal distortion measure for binary document images, IEEE Signal Processing Letters, 11(2), 228-231. 
15. MacQueen, J. et al. (1967). Some methods for classification and analysis of multivariate observations. In Conference Fifth Berkeley Symposium on Mathematical Statistics and Probability (pp. 281-297).

16. Mirjalili, S. et al. (2017). Salp Swarm Algorithm: A bio-inspired optimizer for engineering design problems, Advances in Engineering Software, 114, 163-191. Elsevier.

17. Mitianoudis, N. \& Papamarkos, N. (2015). Document image binarization using local features and Gaussian mixture modeling, Image and Vision Computing, 38, 33-51. Elsevier.

18. Moghaddam, R. F. \& Nafchi, H. Z. (2013). Objective evaluation of binarization methods, MATLAB Central File Exchange [online]. $<\mathrm{http}: / / \mathrm{www}$. mathworks. com/ matlabcentral/fileexchange/27652>.

19. Ntirogiannis, K., Gatos, B. \& Pratikakis, I. (2008). An objective evaluation methodology for document image binarization techniques. In The Eighth IAPR International Workshop on Document Analysis Systems, DAS'08 (pp. 217-224).

20. Otsu, N. (1979). A threshold selection method from gray-level histograms, IEEE Transactions on Systems, Man, and Cybernetics, 9(1), 62-66. IEEE.

21. Paredes, R., Kavallieratou, E. \& Lins, R. D. (2010). ICFHR 2010 Contest: Quantitative evaluation of binarization algorithms. In 2010 International Conference on Frontiers in Handwriting Recognition (ICFHR) (pp. 733-736).

22. Pratikakis, I. et al. (2016). ICFHR 2016 Handwritten Document Image Binarization Contest (H-DIBCO 2016). In $201615^{\text {th }}$ International Conference on Frontiers in Handwriting Recognition (ICFHR) (pp. 619-623).

23. Pratikakis, I., Gatos, B. \& Ntirogiannis, K. (2010). H-DIBCO 2010 - Handwritten
Document Image Binarization Competition. In 2010 International Conference on Frontiers in Handwriting Recognition (ICFHR) (pp. 727-732).

24. Sauvola, J. \& Pietikäinen, M. (2000). Adaptive document image binarization, Pattern Recognition, 33(2), 225-236. Elsevier.

25. Sayed, G. I., Khoriba, G. \& Haggag, M. H. (2018). A novel chaotic salp swarm algorithm for global optimization and feature selection, Applied Intelligence, 1-20. Springer.

26. Serapião, A. B. S. et al. (2016). Combining K-Means and K-Harmonic with Fish School Search Algorithm for data clustering task on graphics processing units, Applied Soft Computing, 41, 290-304. Elsevier.

27. Sokolova, M. \& Lapalme, G. (2009). A systematic analysis of performance measures for classification tasks, Information Processing \& Management, 45(4), 427-437. Elsevier.

28. Tavazoei, M. S. \& Haeri, M. (2007). Comparison of different one-dimensional maps as chaotic search pattern in chaos optimization algorithms, Applied Mathematics and Computation, 187(2), 1076-1085. Elsevier.

29. Vo, Q. N. et al. (2018). Binarization of degraded document images based on hierarchical deep supervised network, Pattern Recognition, 74, 568-586. Elsevier.

30. Wolpert, D. H. \& Macready, W. G. (1997). No free lunch theorems for optimization, IEEE Transactions on Evolutionary Computation, 1(1), 67-82. IEEE.

31. Young, D. P. \& Ferryman, J. M. (2005). Pets metrics: On-line performance evaluation service. In Joint IEEE International Workshop on Visual Surveillance and Performance Evaluation of Tracking and Surveillance (VS-PETS) (pp. 317-324). 
